\title{
Una Tarea de Estimación de Colisiones y Tiempo de Llegada: Evidencias Preliminares de Fiabilidad y Validez ${ }^{1}$
}

\author{
Soledad Susana López², Rubén Daniel Ledesma², Silvana Andrea Montes, María Isabel Introzzi \\ Universidad Nacional de Mar del Plata, Argentina
}

\section{RESUMEN}

Este trabajo analiza algunas propiedades psicométricas de una tarea estimativa de la trayectoria de objetos móviles. Este tipo de tarea suele utilizarse como parte de las baterías de evaluación de conductores en muchos países, pero son escasas las evidencias disponibles sobre validez. Sesenta participantes (treinta conductores profesionales y treinta de población general) completaron la prueba en dos ocasiones. Los resultados fueron positivos en algunos aspectos (e.g., consistencia interna), pero también muestran la necesidad de realizar algunos cambios y mejorías. Se requieren más estudios que ofrezcan evidencias externas de validez para este tipo de tarea.

Palabras clave: Evaluación; Conductores, Tareas Experimentales, Validez.

\section{RESUMO - Uma tarefa de estimativa de colisões e tempo de chegada: evidências preliminares de confiabilidade e} validade

Este trabalho analisa algumas propriedades psicométricas de uma tarefa na estimativa da trajetória de objetos móveis. Esse tipo de tarefa costuma ser utilizado como parte das baterias na avaliação de condutores em muitos países, mas são escassas as evidências disponíveis sobre a validade. Sessenta participantes (30 condutores profissionais e 30 da população geral) completaram a prova em duas ocasiões. Os resultados foram positivos em alguns aspectos (e.g., consistência interna), mas também mostram a necessidade de realizar mudanças e melhorias na prova. Requer-se mais estudos que ofereçam evidências externas que dê validade para esse tipo de tarefa. Palavras-chaves: avaliação, motoristas, tarefas experimentais, validade.

ABSTRACT - A Time-to-contact and Collision Estimation Task: Preliminary Evidences of Reliability and Validity This study analyzes some psychometric properties of a Time-to-contact and Collision Estimation Task. This type of measure is often used as part of driver testing in many countries. However, available evidences of validity are scarce. Sixty participants (split evenly between professional drivers and general population) completed the task on two occasions. The results were positive in some respects (e.g., internal consistency), but also show the need for changes and improvements in the task design. Further studies are needed to support the use of this type of measures.

Keywords: Testing; Drivers, Experimental Tasks; Validity.

\section{Introducción}

La Psicología del Tránsito es un área relativamente joven en Latinoamérica, con desarrollos incipientes en distintos temas (Ledesma, Poó, \& Montes, 2011). Dentro del campo, la construcción de instrumentos de medición resulta clave para la investigación y también para la evaluación de conductores, como base para la toma de decisiones que pueden impactar sobre la vida de las personas (Fernández-Liporacce, Cayssials, \& Pérez, 2009). La calidad de los test psicológicos utilizados en la evaluación de conductores es un tema controvertido, especialmente la validez predictiva de estos instrumentos (e.g., Groeger, 2003; Silva \& Alchieri, 2011). En efecto, las evidencias de validez tienden a ser inconsistentes, sobre todo cuando estas herramientas se utilizan en contextos de acreditación de conductores.

Si bien existen diferentes técnicas de evaluación del desempeño de conductores (Porter, 2011), las tareas informatizadas constituyen una modalidad cada vez más frecuente. La informatización posee la ventaja de incrementar la precisión de las puntuaciones, automatizar la obtención de resultados y generar mayor motivación de respuesta en los sujetos. Muchos países implementan tareas computarizadas en sus procesos de otorgamiento o actualización de licencias. Existen opciones para evaluar diferentes variables, que van desde medidas de tiempo de reacción simple hasta medidas 
de variables más complejas, como la percepción de peligrosidad (e.g., Vlakveld, 2014). Más allá de que su uso es cada vez más frecuente, las evidencias de fiabilidad y validez suelen ser muy escasas.

Entre las medidas más habituales encontramos un sub-conjunto de tareas focalizadas en la percepción y anticipación de trayectorias de objetos móviles. El antecedente más remoto para este tipo de tareas es el Speed Anticipation Reaction Test de Maruyama y Kitamura (1961), que aún en la actualidad es utilizada en varios países. El dispositivo original consistía en la presentación de un objeto móvil de forma esférica, que se desplazaba de manera rectilínea con una trayectoria visible en la mayor parte de su recorrido. En el tramo final del trayecto, el objeto se ocultaba mediante una franja que se interponía verticalmente. El participante debía determinar el instante en que el móvil reaparecería nuevamente al superar la franja o zona de ocultación. En síntesis, se debía seguir la trayectoria del objeto y anticipar el momento de llegada a un punto, luego de haber sido invisibilizado. Actualmente, existen versiones de esta tarea integradas en distintas baterías de evaluación. Por ejemplo, el Sistema de Información de Evaluación Visual, Auditiva, Mental y de Coordinación Motriz (AGX products, 2009) y la batería Asde-Driver Test N-845 (Equipo Psicotécnico Asde driver test N-845, s.f) incluyen el mencionado Speed Anticipation Reaction Test. El Viena System Test (Bauer, Guttmann, Trimmel, Leodolter, \& Leodolter, s.f) también incluye una versión de esta tarea denominada Test ZBA.

Read, Ward y Parkes (2001) desarrollaron una medida de estimación de la trayectoria de un objeto móvil bajo una tarea de atención dividida. En esta tarea se presentan dos automóviles (A y B) que se dirigen hacia el mismo punto, partiendo de distintos lugares. El sujeto debe: (a) estimar el momento en que el automóvil A llega al punto de intersección; (b) estimar si dadas esas trayectorias ambos móviles colisionarán; y (c) identificar en el campo visual eventos relevantes y potencialmente peligrosos que puedan requerir consideración. Se realizaron cuatro estudios para determinar si la tarea era sensible a los efectos de la edad y el deterioro cognitivo, encontrando resultados favorables. Se obtuvieron evidencias sobre su capacidad para discriminar conductores seguros y no seguros, así como la presencia de deterioro cognitivo. También se encontraron correlaciones con medidas de rendimiento en un simulador de conducción.

Un punto débil de este tipo de tareas es que no parece existir acuerdo sobre las definiciones teóricas, los procesos que evalúan y cómo estos se relacionan con una conducción segura. En español suele hablarse de medidas de "Velocidad de Anticipación" (posiblemente por traducción derivada del nombre de la prueba Speed Anticipation Reaction Test), pero la definición de esta expresión suele ser ambigua. Según Calleja, Alía, \& Díaz (2009) este tipo de tareas evalúa la capacidad para percibir velocidades, así como también otros aspectos cognitivos esenciales para una correcta conducción, como el autocontrol. En ocasiones, la velocidad de anticipación se ha definido sólo de modo operativo, como la capacidad de un sujeto para discriminar correctamente la trayectoria, la velocidad y la ubicación de un móvil en un momento dado (Sainz, 1991; en Ramos Alía, 2002). Por su lado, los autores del ZBA se refieren a la capacidad del individuo para imaginar el efecto de un movimiento y estimar correctamente el movimiento de objetos en el espacio. No obstante, tampoco abundan en definiciones o en explicaciones complementarias sobre el marco teórico de esta medida. En síntesis, no se dispone de definiciones teóricas claras sobre los procesos cognitivos involucrados en la tarea, lo cual impacta negativamente sobre los estudios de validez de constructo.

Podría considerarse que la precisión de las definiciones teóricas no es algo tan importante en el ámbito aplicado, donde en definitiva se trata de justificar la utilidad práctica de los instrumentos (por ejemplo, su capacidad para predecir un mayor riesgo de siniestros). El problema es que tampoco se cuenta con evidencias suficientes que avalen su utilidad práctica. Incluso para algunas de las baterías de evaluación más utilizadas y difundidas, las pruebas no siempre presentan evidencias suficientes de fiabilidad y validez. Por ejemplo, es curioso observar la falta de estudios de validez para el ZBA, según se explicita en la propia documentación de usuario de la prueba (Bauer, Guttmann, Trimmel, Leodolter, \& Leodolter, s.f).

Parece claro que se requieren más estudios e investigaciones sobre la validez de las tareas mencionadas antes de poder avanzar al campo de la evaluación. En este contexto, nos hemos interesado en el desarrollo de una prueba que integra las tareas de velocidad de anticipación y de estimación de colisiones (siguiendo a Read, Ward, \& Parkes, 2001). La prueba propuesta posee las siguientes características básicas: (a) evalúa simultáneamente tiempo de llegada y anticipación de colisiones; (b) ofrece flexibilidad para configurar distintos parámetros de la tarea, como la velocidad de los objetos y las áreas de invisibilidad; (c) brinda informes automáticos de resultados; y (d) guarda los datos en un formato que puede ser fácilmente exportable a procesadores estadísticos u otros programas.

Puesto que se trata de una tarea nueva, el presente trabajo se propuso estudiar algunas de sus propiedades psicométricas. En esta etapa, nos hemos focalizado en su fiabilidad y estructura interna. Se espera que el trabajo aporte evidencias para un análisis crítico de estos instrumentos e incentive el desarrollo de nuevas líneas de investigación en el área.

\section{Método}

\section{Participantes}

Se trabajó con un muestreo casual de $n=60$ participantes, 30 conductores profesionales de taxi y remise, y 
30 participantes de población general. Ambos grupos estuvieron equiparados por edad y sexo. Los participantes de población general incluyeron trabajadores de distintas áreas (e.g: áreas de servicios varios, mantenimiento, atención al público, ventas, coordinación de áreas, comercio, etc.). La edad de los sujetos fluctuó entre 28 y 70 años $(\mathrm{M}=46,78$; $\mathrm{DS}=12,5) . \mathrm{El} 96,7 \%$ de la muestra fueron hombres. Previamente, se reclutó una muestra piloto de $n=20$ participantes para realizar ajustes a la tarea.

\section{Instrumento}

\section{Descripción General de la Tarea}

La tarea se denomina Tarea de precisión en la estimación de trayectorias y tiempos de colisión de objetos móviles; y está integrada a la plataforma TAC (Tareas de Autorregulación

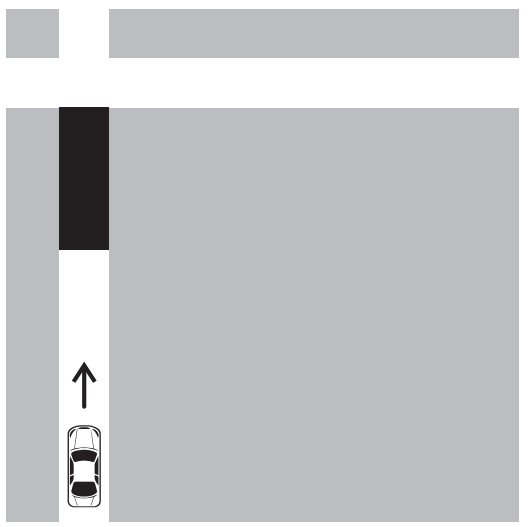

Figura 1. Bloque 1 (estimación de trayectorias)
Cognitiva, Introzzi \& Canet-Juric, 2014; Introzzi, Canet-Juric, Montes, López, \& Mascarello, 2015). Está compuesta por 3 bloques: uno de 9 ensayos y dos de 12, con un ensayo de prueba en cada uno. La duración total de la prueba es de aproximadamente 10 minutos.

El Bloque 1 evalúa la precisión en la estimación de trayectorias y "tiempo de llegada" de un automóvil a un punto determinado. El participante observa un automóvil que aparece debajo de la pantalla y se mueve hacia arriba a una velocidad constante (ver Figura 1). En un momento, el automóvil desaparece pasando por debajo de un "puente" (zona de invisibilidad del automóvil representada por un rectángulo de color negro). La tarea del participante consiste en presionar la barra espaciadora en el momento en que estime que el automóvil llegará a la intersección.

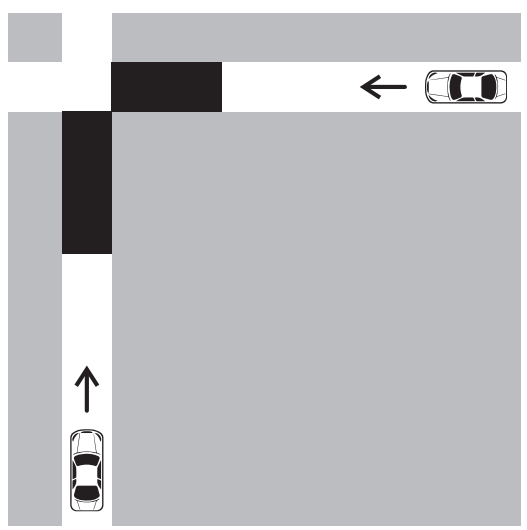

Figura 2. Bloque 2

(anticipación de colisiones)
El Bloque 2 evalúa la habilidad para anticipar la colisión (ver Figura 2). El participante observa la trayectoria de dos automóviles partiendo de diferentes puntos hacia la misma intersección. La tarea consiste en determinar si, dadas las velocidades y trayectorias observadas, los móviles podrían colisionar. Por último, en el Bloque 3 el participante debe realizar simultáneamente ambas tareas.

Los objetos móviles están configurados a diferentes velocidades, lo cual incide en el nivel de dificultad de cada bloque. Las respuestas del participante en cada ensayo se guardan automáticamente, en un formato fácilmente exportable a procesadores estadísticos u otros programas. Se almacenan indicadores sobre precisión en la estimación de los tiempos de llegada y porcentajes de acierto en la anticipación de colisiones.

\section{Diseño Inicial de la Tarea, Criterios y Prueba Piloto}

Siguiendo la literatura previa (Maruyama \& Kitamura, 1961; Read, Ward, \& Parkes, 2001), se tomaron como base para el diseño una serie de criterios que suelen estar presentes en este tipo de tareas. En primer lugar, se buscó que los ensayos presentaran tres fases consecutivas: (a) una fase de observación, es decir un tiempo inicial en el que el participante observa el movimiento del móvil, (b) una fase de estimación o de apreciación de la velocidad, donde el móvil queda oculto por ingresar al área de invisibilidad, y (c) una fase de respuesta, donde el sujeto tiene que reaccionar ajustando su acción a la velocidad del móvil observado. Uno de los requisitos para elaborar la prueba es que la fase de observación no sea menor a la fase de estimación (en el sentido de la trayectoria recorrida), y que la dirección de desplazamiento del móvil sea la misma en ambas fases. Este criterio es importante pues permite que el sujeto pueda prolongar mentalmente una trayectoria (e.g., la distancia total del punto de salida a la esquina es de 480 píxeles, con lo cual la longitud máxima establecida para el área de invisibilidad fue de 200 píxeles). Por otro lado, se buscó que el movimiento fuera continuo, sin intermitencias que pudieran favorecer una estimación por conteo, en lugar de una estimación de velocidad. Por último, la tarea debía tener varios ensayos con distintas velocidades del vehículo. 
Posteriormente, se establecieron distintos valores tanto para la velocidad de los automóviles como para la longitud o tamaño del área de invisibilidad. Se consideró el nivel de dificultad en relación a diferentes criterios internos (e.g., la velocidad de los móviles, la longitud del área de invisibilidad y la diferencia entre la velocidad de ambos vehículos). Se supuso que, a mayor velocidad y tamaño del área de invisibilidad, mayor dificultad del ensayo. Asimismo, se hipotetizó que la dificultad se incrementaría en la medida en que las velocidades de ambos vehículos fueran más similares.

En cada ensayo se configuró una velocidad y trayectoria constante. Para determinar las diferentes velocidades se estableció un rango de 2 a 8 (e.g., la velocidad n ${ }^{\circ}$ 2 fue posteriormente descartada por considerarse muy lenta). Se buscó que las velocidades de ambos vehículos no difirieran demasiado, para no generar ensayos muy fáciles. La velocidad se representa en la cantidad de pixeles por cada 50 milisegundos que recorre el automóvil. Por ejemplo, una velocidad de '5' es igual a la cantidad de píxeles que se mueve en ese tiempo. Con respecto al área de invisibilidad, es importante considerar las dimensiones totales de la pantalla $(600 \times 600)$ y lo que representa cada configuración, en función de la totalidad. Por ejemplo, un área de invisibilidad de 100 píxeles representa 1/6 del mapa. La Tabla 1 muestra los niveles de dificultad posibles para las variables "Velocidad" y "Área de invisibilidad".
Tabla 1

Niveles de Dificultad para las Variables Velocidad y Área de invisibilidad

\begin{tabular}{lccc}
\hline \multicolumn{1}{c}{ Nivel de Dificultad } & Baja & Media & Alta \\
\hline Velocidad & $3-4$ & $5-6$ & $7-8$ \\
Área de invisibilidad & 100 & 150 & 200 \\
\hline
\end{tabular}

En los bloques 2 y 3 , si el automóvil 1 y el 2 colisionan es debido a que se mueven a idénticas velocidades, en cambio si no colisionan es porque las velocidades son distintas. La tarea es más difícil cuando la diferencia entre las velocidades de ambos vehículos es menor. En el Anexo se muestra la configuración final de los ensayos según velocidades y áreas de invisibilidad.

El diseño inicial de la tarea quedó conformado por 3 bloques, compuestos por 6,9 y 9 ensayos respectivamente. Se administró a una prueba piloto de $n=20 \mathrm{su}-$ jetos, y en función de los resultados se realizaron ajustes. Principalmente, se buscó incrementar la dificultad de los ensayos en donde la mayoría de los participantes obtenían aciertos. Para el Bloque 3, se configuraron las mismas áreas de invisibilidad que para el Bloque 2. Por lo tanto, el Bloque 2 y el Bloque 3 son equivalentes (se cambió sólo la velocidad). Por último, se agregaron tres ensayos en cada bloque. De esta forma, la versión definitiva quedó conformada por 3 bloques, compuestos por 9 , 12 y 12 ensayos respectivamente.

Tabla 2

Estructura Definitiva de la Tarea

\begin{tabular}{|c|c|c|}
\hline & $\begin{array}{c}\text { Cantidad } \\
\text { de ensayos }\end{array}$ & Consignas \\
\hline Bloque 1 & 9 & $\begin{array}{l}\text { "En esta tarea vas a tener que estimar movimientos con la mayor precisión posible. En } \\
\text { esta primera parte vas a ver un automóvil que aparece abajo en la pantalla y se mueve } \\
\text { hacia arriba a una velocidad constante. En un momento, el automóvil va a desaparecer, } \\
\text { ya que pasará por debajo de un puente. Tu tarea es presionar la barra espaciadora cuando } \\
\text { creas que el automóvil va a llegar a la esquina (línea roja). Tanto la longitud del puente } \\
\text { como la velocidad del automóvil, pueden cambiar a través de los diferentes ensayos." }\end{array}$ \\
\hline Bloque 2 & 12 & $\begin{array}{l}\text { "Ahora vas a observar dos automóviles, que se dirigen desde diferentes puntos, hacia } \\
\text { la misma esquina. En un momento, ambos van a desaparecer, ya que cada uno de ellos } \\
\text { pasará por debajo de un puente. A continuación se te preguntará si ambos vehículos } \\
\text { van a colisionar o no siguiendo esa misma trayectoria y velocidad." }\end{array}$ \\
\hline Bloque 3 & 12 & $\begin{array}{l}\text { "Ahora vas a realizar las dos tareas anteriores en forma simultánea, (a) vas a observar } \\
\text { la trayectoria de un automóvil en dirección ascendente, que luego va a desaparecer. Tu } \\
\text { tarea va a ser presionar la barra espaciadora en el momento en qué crees que el auto- } \\
\text { móvil alcanzará la esquina, (b) a su vez, vas a observar un segundo automóvil que se } \\
\text { dirige desde otro punto hacia la misma esquina, luego va a desaparecer. Después de } \\
\text { que presiones la barra espaciadora, se te va a preguntar si los vehículos colisionan o no } \\
\text { siguiendo esa misma trayectoria y velocidad." }\end{array}$ \\
\hline
\end{tabular}

\section{Medidas}

La tarea arroja diferentes tipos de resultados para cada ensayo y por bloques. A los efectos de este trabajo, se consideran dos índices básicos de desempeño: (a)
Distancias al Punto de Llegada y (b) Precisión en la Predicción de Colisiones.

Distancias al Punto de Llegada. Estas medidas se calculan para cada ensayo en los Bloques 1 y 3 , donde el 
sujeto tiene que estimar los momentos en que el móvil alcanzaría un punto de llegada. Indican las diferencias absolutas entre el momento estimado y el momento de llegada objetivo, y se expresan en milisegundos. Mayores tiempos indican peor desempeño en la tarea. Además de las medidas por ensayo, se calculan las medidas promedio por bloque y total. Para simplificar, hablaremos de "Distancia Bloque 1", "Distancia Bloque 3", "Distancia Total" para referirnos a estas medidas promedio. Nuevamente, mayores valores indican peor rendimiento.

Precisión en la Predicción de Colisiones. Estas medidas se calculan para cada ensayo en los Bloques 2 y 3 , donde el sujeto tiene que estimar la posibilidad de una colisión. Indican simplemente aciertos o desaciertos en la predicción. Además de las medidas por ensayo, se calcula la proporción de aciertos por bloque y total. Para simplificar, hablaremos de "Precisión Bloque 2", "Precisión Bloque 3", y "Precisión Total". En este caso, mayores valores indican mejor rendimiento.

\section{Procedimiento}

Todos los participantes fueron evaluados en dos momentos de un mismo día, con una diferencia de entre 8 y 12 horas entre las tomas (antes y después de la jornada laboral). Los sujetos fueron contactados e invitados a participar del estudio utilizando un muestreo casual en red. Las tareas se administraron previo consentimiento informado, garantizando la confidencialidad de los resultados. Las condiciones de administración de la prueba fueron equivalentes para todos los participantes, quienes respondieron en un lugar tranquilo y sin distracciones, con la presencia de un investigador.

\section{Análisis de Datos}

Se realizaron los siguientes análisis de datos: a) análisis de estadísticos descriptivos (media, desvío estándar, mínimos y máximos), para las variables Distancias al punto de llegada y Precisión en la predicción de Colisiones en ambos momentos; b) prueba $t$ para muestras relacionadas, para comparar el desempeño del participante en los distintos bloques de la tarea, en los dos momentos. Se realizaron análisis de significación de esas diferencias y su magnitud o tamaño del efecto. A su vez, se realizaron análisis de correlación entre ambos momentos, para la variable Precisión en la Predicción de Colisiones y para la variable Distancias al punto de llegada; (c) finalmente, se realizó una prueba t para muestras independientes, para comparar el desempeño de los participantes en ambos grupos (población general y conductores profesionales).

\section{Resultados}

\section{Análisis Descriptivos}

La Tabla 3 presenta los descriptivos para la variable distancias al punto de llegada en ambos momentos. Se observan diferencias a nivel descriptivo entre el bloque $1 \mathrm{y}$ 3 (peor desempeño en el bloque más difícil). Los tamaños del efecto son bajos, aunque en el sentido de lo esperado (M1: Cohen's $d:-0,20 ;$ M2: Cohen's $d:-0,13$ ), pero las diferencias no resultan significativas (momento 1: $t(59)=-1,51, p=0,13$; momento dos: $t(59)=0,99$, $p=0,32$ ). Tampoco se detectaron diferencias significativas para un mismo bloque en los dos momentos (bloque 1: $t(59)=0,06, p=0,95$; y bloque 3: $t(59)=0,9, p=0,36)$. Este resultado sugiere que no habría efecto de aprendizaje entre ambas tomas. Un análisis de medidas repetidas confirma todos los resultados anteriores. Para realizar estos cálculos se consideraron todos los ensayos de los bloques 1 y 3 .

La Tabla 4 presenta los descriptivos para la variable Precisión en la Predicción de Colisiones en ambos momentos. Prácticamente no se observan diferencias entre bloques 2 y 3 . Las diferencias no son significativas (momento 1: $t(59)=-0,57, p=0,56$; momento dos: $t(59)=0,01$ $, p=0,99)$. A su vez, se observó que los tamaños del efecto son bajos (M1: Cohen's $d$ : $-0,075, \mathrm{M} 2$ : Cohen's $d$ : $0,01)$. Tampoco se detectaron diferencias significativas para un mismo bloque en los dos momentos (bloque 2: $t(59)=1,17, p=0,24$; $y$ bloque 3: $t(59)=1,06, p=0,29)$. Para realizar estos cálculos, no se consideraron los 4 ítems que fueron eliminados previamente (ver apartado fiabilidad).

Tabla 3

Descriptivos para la Variable Distancias al punto de llegada en Ambos Momentos

\begin{tabular}{|c|c|c|c|c|c|c|c|c|}
\hline & \multicolumn{2}{|c|}{ Media } & \multicolumn{2}{|c|}{ DS } & \multicolumn{2}{|c|}{ Min } & \multicolumn{2}{|c|}{ Max } \\
\hline & M1 & M2 & M1 & M2 & M1 & M2 & M1 & M2 \\
\hline Distancia / Bloque 1 & 358,75 & 356,97 & 244,39 & 234,92 & 97 & 39 & 1215 & 1178 \\
\hline Distancia / Bloque 3 & 404,37 & 380,98 & 244,51 & 210,69 & 122 & 100 & 1372 & 1215 \\
\hline
\end{tabular}

${ }^{*}$ M1=Momento 1, M2=Momento 2

Tabla 4

Descriptivos para la Variable Precisión en la Predicción de Colisiones en Ambos Momentos

\begin{tabular}{|c|c|c|c|c|c|c|c|c|}
\hline & \multicolumn{2}{|c|}{ Media } & \multicolumn{2}{|c|}{ DS } & \multicolumn{2}{|c|}{ Min } & \multicolumn{2}{|c|}{ Max } \\
\hline & M1 & M2 & M1 & M2 & M1 & M2 & M1 & M2 \\
\hline Precisión / Bloque 2 & 0,74 & 0,78 & 0,23 & 0,21 & 0,13 & 0,13 & 1 & 1 \\
\hline Precisión / Bloque 3 & 0,75 & 0,78 & 0,23 & 0,21 & 0,13 & 0,25 & 1 & 1 \\
\hline
\end{tabular}




\section{Estimaciones de Fiabilidad}

La Tabla 5 resume las estimaciones de fiabilidad para las principales medidas de la tarea. En cuanto a la variable Distancias al punto de llegada, los resultados son satisfactorios para los dos bloques en ambos momentos ( $\alpha$ de Cronbach $>0,85$ en todos los casos). En relación a las medidas de Precisión en la Predicción de Colisiones, los análisis de fiabilidad indicaron la necesidad de eliminar varios ensayos $(2,3,6$ y 8$)$ por presentar varianza $=0 \mathrm{y} / \mathrm{o}$ índices muy bajos de discriminación. Al eliminar estos ensayos, se obtienen resultados satisfactorios para la medida global (KR-20 >0,80 en ambos momentos), pero no tanto para las medidas parciales (por bloque). Por otro lado, al analizar la correlación entre ambos momentos se obtiene $r=0,49$ para la variable Precisión en la Predicción de Colisiones y $r=0,66$ para la variable Distancias al punto de llegada. Esto indicará mayor estabilidad en la medida de Distancias, en comparación con la de Precisión.

Tabla 5

Estimaciones de Fiabilidad por Bloques y Totales

\begin{tabular}{lcccc}
\hline & Descripción & Estimación & Primera toma & Segunda toma \\
\hline $\begin{array}{l}\text { Distancias al } \\
\text { punto de llegada }\end{array}$ & Bloque 1 & Alfa de Cronbach & 0,89 & 0,91 \\
& Bloque 3 & Alfa de Cronbach & 0,89 & 0,86 \\
& Total & Alfa de Cronbach & 0,93 & 0,93 \\
Precisión en la & Bloque 2 & & & 0,63 \\
Predicción de & Bloque 3 & KR-20 & 0,65 & 0,60 \\
Colisiones & Total & KR-20 & 0,66 & 0,81 \\
\hline
\end{tabular}

Las Tablas 6 y 7 muestran los descriptivos para los ensayos que finalmente componen las medidas de Distancias al punto de llegada y Precisión en la Predicción de Colisiones, respectivamente. Para la variable Distancias al punto de llegada los ensayos que resultaron ser más difíciles (media superior a 400) fueron: para el bloque 1, ensayos 1, 4 y 7, para el bloque 3, ensayos 3, 4, 5 y 6. Se observa que aquellos ensayos que presentaron mayor dificultad para los participantes fueron aquellos que estaban configurados con mayor área de invisibilidad (ver Anexo).

Tabla 6

Descriptivos por Ensayo Variable Distancias al Punto de Llegada*

\begin{tabular}{|c|c|c|c|c|c|c|c|c|}
\hline \multirow{2}{*}{ Bloque 1} & \multicolumn{4}{|c|}{ Momento 1} & \multicolumn{4}{|c|}{ Momento 2} \\
\hline & Media & Ds & Min & Max & Media & Ds & Min & $\operatorname{Max}$ \\
\hline E.1. Distancia & 425,58 & 469,88 & 0 & 1968 & 334,35 & 297,21 & 0 & 1661 \\
\hline E.2. Distancia & 288,48 & 291,34 & 0 & 1181 & 263,23 & 270,24 & 0 & 1054 \\
\hline E.3. Distancia & 397,48 & 321,88 & 0 & 1341 & 371,11 & 301,58 & 48 & 1422 \\
\hline E.4. Distancia & 604,81 & 714,15 & 0 & 5161 & 526,96 & 362,45 & 48 & 1500 \\
\hline E.5. Distancia & 258,15 & 218,23 & 26 & 927 & 187,91 & 159,68 & 0 & 712 \\
\hline E.6. Distancia & 348,28 & 279,68 & 0 & 1128 & 341,38 & 252,08 & 0 & 1214 \\
\hline E.7. Distancia & 374,20 & 285,15 & 48 & 1176 & 403,11 & 292,42 & 0 & 1314 \\
\hline E.8. Distancia & 318,10 & 251,01 & 0 & 975 & 290,93 & 261,87 & 0 & 1087 \\
\hline E.9. Distancia & 313,73 & 243,71 & 26 & 841 & 330,40 & 272,20 & 0 & 1122 \\
\hline Bloque 3 & Media & Ds & Min & $\operatorname{Max}$ & Media & Ds & Min & $\operatorname{Max}$ \\
\hline E.1. Distancia & 252,98 & 255 & 0 & 1367 & 227,46 & 242,34 & 0 & 1286 \\
\hline E.2. Distancia & 353,63 & 355,72 & 0 & 1188 & 371,55 & 414,04 & 0 & 1649 \\
\hline E.3. Distancia & 440,86 & 404,06 & 48 & 1820 & 458,05 & 371,49 & 48 & 1750 \\
\hline E.4. Distancia & 534,41 & 393,69 & 0 & 1673 & 526,96 & 362,45 & 48 & 1500 \\
\hline E.5. Distancia & 472,36 & 399,23 & 0 & 1943 & 506,08 & 366,63 & 50 & 1427 \\
\hline E.6. Distancia & 608,31 & 509,69 & 0 & 1924 & 626,28 & 564,24 & 0 & 2064 \\
\hline E.7. Distancia & 295,91 & 232,15 & 0 & 973 & 252,41 & 239,81 & 0 & 1008 \\
\hline E.8. Distancia & 318,78 & 264,54 & 40 & 992 & 306,70 & 265,80 & 0 & 1185 \\
\hline E.9. Distancia & 353,71 & 263,02 & 0 & 1080 & 321,35 & 248,60 & 34 & 1332 \\
\hline E.10. Distancia & 374,65 & 308,85 & 42,00 & 1281 & 290,08 & 242,45 & 40 & 1249 \\
\hline E.11. Distancia & 386,78 & 320,57 & 0 & 1288 & 322,76 & 241,92 & 0 & 829 \\
\hline E.12. Distancia & 386,15 & 292,75 & 0 & 1176 & 357,08 & 270,60 & 0 & 1128 \\
\hline
\end{tabular}


Tabla 7

Descriptivos por Ensayo Variable Precisión en la Predicción de Colisiones*

\begin{tabular}{|c|c|c|c|c|}
\hline ? מונמקlPl & \multicolumn{2}{|c|}{ Momento 1} & \multicolumn{2}{|c|}{ Momento 2} \\
\hline Droque 2 & Media & Ds & Media & Ds \\
\hline E.1. Precisión & 0,82 & 0,39 & 0,90 & 0,30 \\
\hline E.4. Precisión & 0,87 & 0,34 & 0,75 & 0,43 \\
\hline E.5. Precisión & 0,60 & 0,49 & 0,85 & 0,36 \\
\hline E.7. Precisión & 0,93 & 0,25 & 0,95 & 0,21 \\
\hline E.9. Precisión & 0,65 & 0,48 & 0,68 & 0,46 \\
\hline E.10. Precisión & 0,86 & 0,34 & 0,83 & 0,37 \\
\hline E.11. Precisión & 0,78 & 0,41 & 0,80 & 0,40 \\
\hline E.12. Precisión & 0,38 & 0,49 & 0,40 & 0,49 \\
\hline Bloque 3 & Media & Ds & Media & Ds \\
\hline E.1. Precisión & 0,85 & 0,36 & 0,90 & 0,30 \\
\hline E.4. Precisión & 0,87 & 0,34 & 0,75 & 0,43 \\
\hline E.5. Precisión & 0,60 & 0,49 & 0,85 & 0,36 \\
\hline E.7. Precisión & 0,93 & 0,25 & 0,95 & 0,21 \\
\hline E.9. Precisión & 0,65 & 0,48 & 0,68 & 0,46 \\
\hline E.10. Precisión & 0,87 & 0,34 & 0,83 & 0,37 \\
\hline E.11. Precisión & 0,78 & 0,41 & 0,80 & 0,40 \\
\hline E.12. Precisión & 0,38 & 0,49 & 0,40 & 0,49 \\
\hline
\end{tabular}

${ }^{*}$ La media se expresa en proporción de aciertos

Para la variable Precisión en la Predicción de Colisiones los ensayos que resultaron ser más difíciles (menor porcentaje de aciertos) fueron tanto como para el bloque 2 como para el bloque 3 , los ensayos 5,9 y 12 , siendo también estos ensayos los que presentaban mayores áreas de invisibilidad. De esta variable el valor mínimo es 0 y el máximo es 1 .

\section{Conductores de Población General us Profesionales}

La Tabla 8 muestra la media y el desvío estándar para los totales en distancias al punto de llegada, en ambos momentos y en ambos grupos. No se observaron diferencias significativas en esta variable en ninguno de los dos momentos (momento 1: $t(58)=-0,50, p=0,82$; momento $2: t(58)=0,27, p=0,90)$.

Tabla 8

Media y Desuío Estándar para los Totales en Variable Distancias al Punto de Llegada, en Ambos Momentos

\begin{tabular}{lccc}
\hline & Grupo & Media & DS \\
\hline \multirow{2}{*}{ Total Distancia en Momento 1 (bloque 1 y 3) } & Profesional & 736,22 & 390,80 \\
& Población general & 791,86 & 473,09 \\
Total Distancia Momento 2 (bloque 1 y 3) & Profesional & 752,03 & 375,44 \\
& Población general & 722,89 & 441,42 \\
\hline
\end{tabular}

La Tabla 9 muestra la media y el desvío estándar para los totales en Precisión en la Predicción de Colisiones, en ambos momentos y en ambos grupos. Las diferencias no resultan significativas (momento 1: $t(58)=-0,50, p=0,83$; momento $2: t(58)=0,27, p=0,90)$. Se realizó un análisis de medidas repetidas con los totales $(\mathrm{b} 2+\mathrm{b} 3)$ en los dos momentos, obteniendo resultados en el mismo sentido.

Tabla 9

Media y Desuío Estándar para los Totales en Variable Precisión en la Predicción de Colisiones, en Ambos Momentos

\begin{tabular}{lccc}
\hline & Grupo & Media & DS \\
\hline \multirow{2}{*}{ Total Precisión en Momento 1 (bloque 2 y 3) } & Profesional & 1,44 & 0,44 \\
& Población general & 1,51 & 0,43 \\
Total Precisión Momento 2 (bloque 2 y 3) & Profesional & 1,60 & 0,32 \\
& Población general & 1,47 & 0,47 \\
\hline
\end{tabular}




\section{Discusión}

En este trabajo propusimos y analizamos una nueva Tarea de estimación de trayectorias y tiempos de colisión de objetos móviles. A continuación, resumimos los resultados, las ventajas y limitaciones, y las posibles líneas para avanzar en el estudio de la tarea.

La experiencia realizada indica como primer aspecto positivo la simplicidad en la administración de la prueba. Los participantes no presentaron dificultades para comprender las consignas ni para ejecutar la tarea. Vale mencionar además que se trata de una tarea breve, que puede administrarse sin recursos o equipamientos especiales, una vez que se instala en una computadora la plataforma en la que funciona la tarea. Sin duda, la simplicidad es uno de los motivos por los cuáles estas tareas son interesantes en la práctica. Además, la técnica ofrece la posibilidad de efectuar cambios en la configuración, es decir de modificar parámetros como consignas, velocidad y el área de invisibilidad en los ensayos que componen cada bloque. Esto implica una importante ventaja respecto a otras herramientas dado que el evaluador puede adaptar los parámetros en función de sus propósitos $\mathrm{u}$ objetivos, aportando cierta flexibilidad al proceso de evaluación.

En cuanto a los resultados sobre la fiabilidad de los puntajes (consistencia interna), estos fueron satisfactorios para las medidas globales de Distancias al punto de llegada y Precisión en la Predicción de Colisiones. Para el caso de la variable Precisión en la Predicción de Colisiones, los puntajes fueron buenos para la medida global, pero luego de eliminar algunos ensayos que no presentaban prácticamente variabilidad (todos o casi todos los sujetos acertaban). Justamente, una de las limitaciones encontradas en esta medida tiene que ver con el bajo nivel de dificultad. Se observaron muchos aciertos e incluso ítems con varianza cero, lo que debe ser mejorado en el futuro. No obstante, también es posible que para otros grupos específicos (e.g., muestras clínicas, adultos mayores o conductores en rehabilitación) sea importante contar con ensayos de baja dificultad que puedan ser sensibles para detectar problemáticas graves de desempeño, evaluar conductores que se encuentran en rehabilitación, etc.
En relación a la dificultad y la validez interna de la prueba, no se observaron diferencias entre los bloques 1 y 3 . Se esperaba un peor rendimiento en este último bloque, pues supone la realización de ambas tareas simultáneamente. A nuestro juicio, los ensayos que evalúan la variable anticipación de colisiones no resultaron lo suficientemente difíciles como para interferir en el desempeño de los sujetos. Entendemos que será necesario lograr configuraciones con mayor nivel de dificultad, que aseguren mayor variabilidad a los ensayos (especialmente en los aciertos y desaciertos en la estimación de colisiones). Una posibilidad sería aumentar la longitud del área de invisibilidad (en la que los automóviles desaparecen del campo visual del participante por un determinado tiempo), ya que observamos que los ensayos más difíciles son aquellos que éstas son mayores. Otra opción podría ser aumentar las velocidades de los automóviles o configurarlas de otra manera. Al igual que en la literatura previa sobre el tema (González Calleja \& Cerro, 1986), observamos que se producen más errores o los tiempos de respuesta aumentan cuando existe una configuración más alta de la velocidad.

Por último, tampoco logramos encontrar diferencias de desempeño entre el grupo de profesionales y de población general, lo que suponíamos podía aportar evidencias de validez para la tarea. Nuevamente, la ausencia de diferencias se podría explicar por la dificultad de la tarea. Quizás los ensayos resultaron demasiado sencillos para la mayoría de los participantes, lo que hizo que el desempeño se equipare, más allá de las diferencias que pudieran existir entre ambos grupos. En todo caso, es claro que se requieren más estudios para obtener evidencias de validez para la tarea, comparando distintas poblaciones, niveles de experticia en la conducción, antecedentes en siniestralidad, etc.

Nuestro estudio muestra la importancia de realizar investigaciones sobre las propiedades psicométricas de los instrumentos, incluso en aquellas tareas de uso extendido. Creemos que resta mucho camino de investigación antes de poder utilizar estas herramientas en contextos de evaluación. En consonancia con otros autores (Silva \& Alchieri, 2007, 2008, 2011), debemos destacar la importancia de contar con más evidencias de validez para las pruebas de evaluación en psicología del tránsito.

\section{Referencias}

AGX products (2009). Test de velocidad de anticipación [instrumento de medida]. Recuperado de de http://www.agxproducts.com/Productos/ Seguridad_Industrial/Bateria_Psicomotriz/Tests/tests_4.html

Bauer, H., Guttmann, G., Trimmel, M., Leodolter, M., \& Leodolter, U. (s.f.). Viena System Test. ZBA Time/Movement Anticipation [Computer software]. Recuperado de http://www.schuhfried.es/vienna-test-system/tests-y-test-sets/todos-los-tests-de-la-a-a-la-z/test/zbatimemovement-anticipation-2/ 
Calleja, F. G., Alía, J. F. R., \& Díaz, J. M. (2009). Análisis crítico de la medida de la velocidad de anticipación en el proceso de evaluación psicológica de conductores en España. Revista de Ciencias Sociales, 3, 1-31.

Equipo Psicotécnico Asde driver test N-845 (sf.). Test de velocidad de anticipación [instrumento de medida]. Asde operators. Recuperado de http://www.generalasde.com/driver-test/

Fernández Liporace, M. M., Cayssials, A., \& Pérez, M. (2009). Curso básico de Psicometría. Buenos Aires: Lugar.

González Calleja, F., \& Cerro, V. J. (1986). Manual del Test de Velocidad de Anticipación KCC, Kelvin, Madrid.

Groeger, J. A. (2003). Testes psicológicos podem predizer habilidade para dirigir? Em R. Risser (Ed.), Estudos Sobre a Avaliação Psicológica de Motoristas (pp. 79-95). São Paulo: Casa do Psicólogo.

Hogan, T. P. (2015). Pruebas psicológicas: Una introducción práctica. Editorial El Manual Moderno.

Introzzi, I., \& Canet Juric, L. (2014). Evaluación de las Funciones Ejecutivas. XVIII Congreso Nacional de Psicodiagnóstico. Asociación Argentina de Estudio e Investigación en Psicodiagnóstico ADEIP. Mar del Plata, Argentina. Recuperado de en www.adeip.org.ar/Congreso2014

Introzzi, I., Canet-Juric, L., Montes, S. A., López, S., \& Mascarello, G. (2015). Procesos Inhibitorios y flexibilidad cognitiva: Evidencia a favor de la Teoría de la Inercia Atencional. International Journal of Psychological Research, 8(2), 61-75.

Ledesma, R. D., Poó, F. M., \& Montes, S. A. (2011). Psicología del tránsito: Logros y desafíos de la investigación. Psiencia: Revista Latinoamericana de Ciencia Psicológica, 3, 108-119.

Maruyama, K., \& Kitamura, S. (1961). Speed anticipation test: A test for discrimination of accident proneness in motor driver. Tohoku Psychological Folia, 20, 13-20.

Porter, B. E. (Ed.). (2011). Handbook of traffic psychology. Academic Press.

Ramos Alía, J. F. (2002). Atención y velocidad de anticipación: Una aproximación crítica al estudio y medida de la atención (tesis doctoral). Universidad Complutense de Madrid, Facultad de Psicología, Departamento de Psicología Evolutiva y de la Educación, Madrid.

Read, N. L., Ward, N. J., \& Parkes, A. M. (2001). Time-to-contact and collision detection estimations as measures of driving safety in old and dementia drivers. Proceedings of the First International Driving Symposium on Human Factors in Driving Assessment, Training and Vehicle Design, Aspen, CO (pp. 240-245).

Silva, F. H. V. C., \& Alchieri, J. C. (2007). Avaliação psicológica da personalidade de condutores: Uma revisão de literatura. Psico-USF, 12, 189-196.

Silva, F. H. V. C., \& Alchieri, J. C. (2008). Revisão das pesquisas brasileiras em avaliação psicológica de habilidades e inteligência de condutores. Estudos de Psicologia (Natal), 13, 57-64.

Tornimbeni, S., Pérez, E., Olaz, F., de Kohan, N. C., Fernández, A., \& Cupani, M. (2008). Introducción a la psicometría. Buenos Aires: Paidós.

Vieira de Cristo e Silva, F. H., \& Alchieri, J. C. (2011). Validade preditiva de instrumentos psicológicos usados na avaliação psicológica de condutores. Psicologia: Teoria e Pesquisa, 26, 695-706.

Vlakveld, W.P. (2014) A comparative study of two desktop hazard perception tasks suitable for mass testing in which scores are not based on response latencies. Transportation Research Part F: Traffic Psychology and Behaviour, 22, 218-231.

Anexo

Configuración Final de los Ensayos Según Velocidad y Área de Invisibilidad

\begin{tabular}{|c|c|c|c|c|c|c|}
\hline \multirow{2}{*}{ Auto 1} & \multicolumn{3}{|c|}{ Velocidad } & \multicolumn{3}{|c|}{ Área de invisibilidad } \\
\hline & Bloque 1 & Bloque 2 & Bloque 3 & Bloque 1 & Bloque 2 & Bloque 3 \\
\hline Ensayo 1 & 3 & 4 & 5 & 100 & 100 & 100 \\
\hline Ensayo 2 & 6 & 4 & 5 & 100 & 100 & 100 \\
\hline Ensayo 3 & 4 & 5 & 4 & 150 & 150 & 150 \\
\hline Ensayo 4 & 4 & 5 & 3 & 200 & 150 & 150 \\
\hline Ensayo 5 & 8 & 3 & 4 & 100 & 200 & 200 \\
\hline Ensayo 6 & 6 & 4 & 5 & 150 & 200 & 200 \\
\hline Ensayo 7 & 5 & 6 & 7 & 200 & 100 & 100 \\
\hline Ensayo 8 & 7 & 6 & 7 & 150 & 100 & 100 \\
\hline Ensayo 9 & 8 & 7 & 6 & 200 & 150 & 150 \\
\hline Ensayo 10 & & 8 & 7 & & 150 & 150 \\
\hline Ensayo 11 & & 7 & 8 & & 200 & 200 \\
\hline Ensayo 12 & & 7 & 8 & & 200 & 200 \\
\hline Auto 2 & & Velocidad & & \multicolumn{3}{|c|}{ Área de invisibilidad } \\
\hline Ensayo 1 & & 4 & 5 & & 100 & 100 \\
\hline Ensayo 2 & & 5 & 4 & & 100 & 100 \\
\hline Ensayo 3 & & 4 & 5 & & 150 & 150 \\
\hline Ensayo 4 & & 5 & 3 & & 150 & 150 \\
\hline Ensayo 5 & & 3 & 4 & & 200 & 200 \\
\hline Ensayo 6 & & 5 & 4 & & 200 & 200 \\
\hline Ensayo 7 & & 6 & 7 & & 100 & 100 \\
\hline Ensayo 8 & & 7 & 6 & & 100 & 100 \\
\hline
\end{tabular}


Anexo (continuación)

Configuración Final de los Ensayos Según Velocidad y Área de Invisibilidad

\begin{tabular}{|c|c|c|c|c|c|c|}
\hline \multirow{2}{*}{ Auto 1} & \multicolumn{3}{|c|}{ Velocidad } & \multicolumn{3}{|c|}{ Área de invisibilidad } \\
\hline & Bloque 1 & Bloque 2 & Bloque 3 & Bloque 1 & Bloque 2 & Bloque 3 \\
\hline Ensayo 9 & & 6 & 7 & & 150 & 150 \\
\hline Ensayo 10 & & 8 & 7 & & 150 & 150 \\
\hline Ensayo 11 & & 7 & 8 & & 200 & 200 \\
\hline Ensayo 12 & & 8 & 7 & & 200 & 200 \\
\hline
\end{tabular}

${ }^{*}$ La velocidad se representa en la cantidad de pixeles por cada 50 milisegundos que recorre el automóvil. El área de invisibilidad, se expresa en píxeles

\section{Sobre os autores}

Soledad Susana López es Licenciada en Psicología por la Universidad Nacional de Mar del Plata. Trabaja en el Instituto de Psicología Básica, Aplicada y Tecnología (IPSIBAT) de la Facultad de Psicología de la Universidad Nacional de Mar del Plata (UNMdP), Argentina. Es Becaria doctoral del Consejo Nacional de Investigaciones Científicas y Técnicas (CONICET) y docente en la Facultad de Psicología de la UNMdP. Es especialista en Psicoterapia Cognitiva (UNMdP-Fundación Aiglé).

Rubén Daniel Ledesma es Doctor en Psicología por la Universidad de Valencia. Trabaja en el Instituto de Psicología Básica, Aplicada y Tecnología (IPSIBAT) de la Facultad de Psicología de la Universidad Nacional de Mar del Plata (UNMdP), Argentina. Es Investigador del Consejo Nacional de Investigaciones Científicas y Técnicas (CONICET) y docente en la Facultad de Ciencias de la Salud y Trabajo Social de la UNMdP.

Silvana Andrea Montes es Doctora en Psicología por la Universidad Nacional de Mar del Plata. Trabaja en el Instituto de Psicología Básica, Aplicada y Tecnología (IPSIBAT) de la Facultad de Psicología de la Universidad Nacional de Mar del Plata (UNMdP), Argentina. Es Investigadora Asistente del Consejo Nacional de Investigaciones Científicas y Técnicas (CONICET) y docente en la Facultad de Psicología de la UNMdP.

María Isabel Introzzi es Doctora en Psicología por la Universidad Nacional de San Luis. Trabaja en el Instituto de Psicología Básica, Aplicada y Tecnología (IPSIBAT) de la Facultad de Psicología de la Universidad Nacional de Mar del Plata (UNMdP), Argentina. Es Investigadora del Consejo Nacional de Investigaciones Científicas y Técnicas (CONICET) y docente en la Facultad de Psicología de la UNMdP. 